\title{
Modulated scattering array antennas for mobile handsets
}

\author{
Qiaowei Yuan $^{1}$, Masaya Ishizu ${ }^{2}$, Qiang Chen ${ }^{2 a)}$, and Kunio Sawaya ${ }^{2}$ \\ ${ }^{1}$ Intelligent Cosmos Research Institute \\ Aramaki Aza Aoba 6-6-05, Aoba-ku, Sendai 980-8579, Japan \\ ${ }^{2}$ Tohoku University \\ Aramaki Aza Aoba 6-6-05, Aoba-ku, Sendai 980-8579, Japan \\ a)chenq@ecei.tohoku.ac.jp
}

\begin{abstract}
A concept of modulated scattering array antenna is proposed based on the modulated scattering technique. The array antenna consists of one antenna element and several modulated scattering elements (MSE). The configuration of the array antenna is very simple because only one branch of the front-end circuit is required. It is shown by experimental investigations that a 3 -element modulated scattering array antenna composed of one $\lambda / 4$ monopole and two $\lambda / 4$ modulated scattering monopoles with $\lambda / 4$ array spacing can provide diversity gain up to $8 \mathrm{~dB}$ diversity gain at $0.1 \%$ of the cumulative distribution function in Rayleigh fading environment. The array antenna is suitable to the mobile handset applications where the space and the cost are strictly limited because of its simple configuration.
\end{abstract}

Keywords: antenna, array antenna, modulation, wireless communications, mobile handset

Classification: Microwave and millimeter wave devices, circuits, and systems

\section{References}

[1] J. H. Winters, "Smart antennas for wireless systems," IEEE Trans. Pers. Commun., pp. 23-27, Feb. 1998.

[2] Tayeb A. Denidni, Dave McNeil, and Gilles Y. Delisle, "Experimental Investigations of a New Adaptive Dual-Antenna Array for Handset Applications," IEEE Trans. Veh. Technol., vol. 52, no. 6, Nov. 2003.

[3] S. C. K. Ko and R. D. Murch, "Compact integrated diversity antenna for wireless communications," IEEE Trans. Antennas Propagat., vol. 49, pp. 954-960, June 2001.

[4] J. H. Richmond, "A Modulated Scattering Technique for Measurement of Field Distribution," IRE Trans. Mirowave Theory Tech., vol. MTT-3, no. 4, pp. 13-15, July 1955. 


\section{Introduction}

Array antennas have been widely studied and used for basestations because they are effective in solving the problems of multipath fading and co-channel interference and improving performance and capacity in wireless communication systems [1]. However, little effort has been made for developing array antennas suitable for mobile handsets, because of the difficulties such as the limited space on the handset to mount an antenna array with sufficiently low spatial correlation, high cost and complexity of the printed board circuits for operating adaptive algorithm $[2,3]$. Therefore, it is required to develop array antennas with simple configurations which are suitable for mobile handsets. In this letter, a new concept of array antennas, which is called modulated scattering array antenna (MSAA), is proposed based on the modulated scattering technique. The configurations of the MSAA are very simple compared with that of the ordinary array antenna. Experiment has been performed to investigate the performance of the MSAA.

\section{Concept of Modulated Scattering Array Antenna}

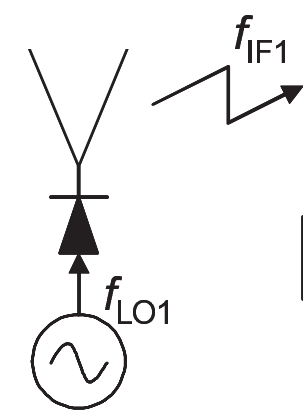

MSE

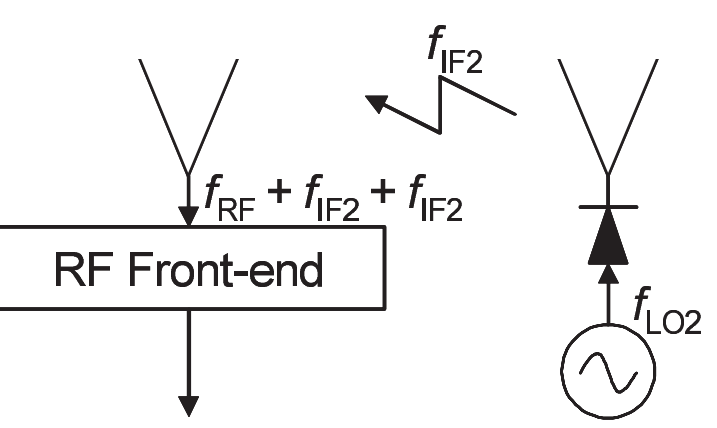

Normal Antenna

\section{MSE}

Fig. 1. The concept of the receiving modulated scattering array antenna.

The concept of the MSAA for reception is shown in Fig. 1. The array antenna consists of one antenna element and several modulated scattering elements (MSEs). Only one branch of receiving circuit is connected to the antenna element, while the MSEs do not have their own receiving circuits. The MSE is a conducting scatter where a nonlinear impedance is loaded and a local signal with low-frequency $f_{\mathrm{LO}}$ is applied to the MSE for modulation [4]. The incident electromagnetic field with frequency $f_{\mathrm{RF}}$ at the MSE position is modulated by the local signal, and the modulated signal with frequency $f_{\mathrm{IF}}$ scattered by the MSE is received by the nearby normal antenna which is connected to a RF receiver. Since the frequency of local signal for each MSE is different, the received signal by the normal antenna and the $\mathrm{RF}$ receiver involves different frequency components corresponding to different array branches including the normal antenna itself and the MSEs. The 
received signal can be used for the adaptive combination since each array branch can be distinguished in the frequency domain.

\section{Experimental investigations}
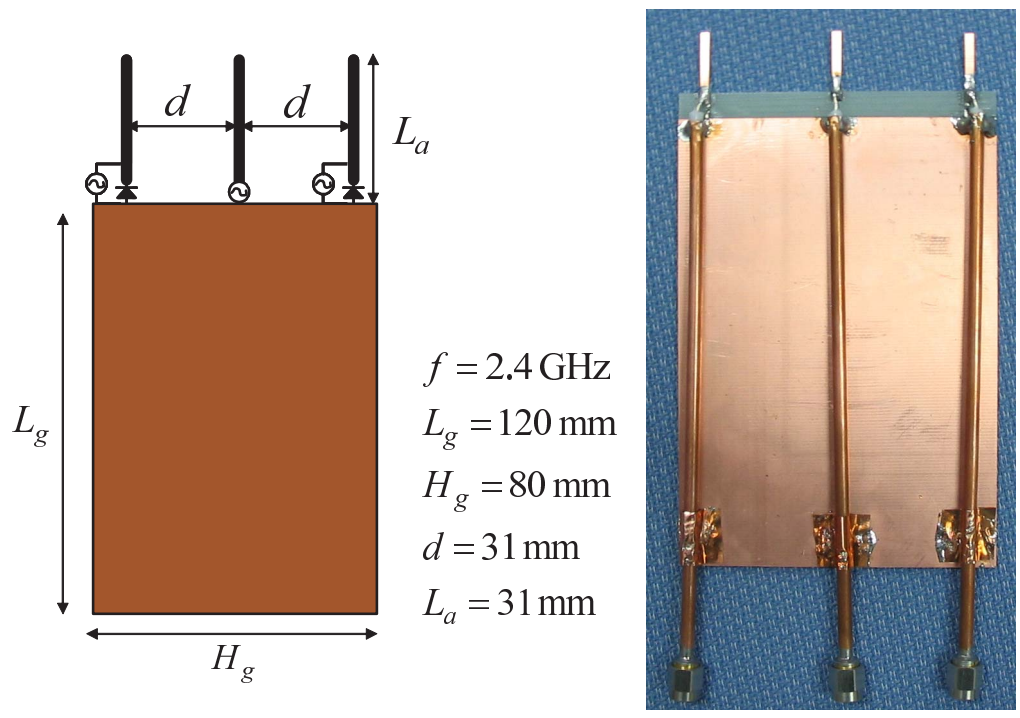

Fig. 2. A 3-element MSAA for handset applications.

3-element MSAA for handset applications shown in Fig. 2 was fabricated for experimental investigation. The array antenna designed at a frequency of $2.4 \mathrm{GHz}$ is composed of three $\lambda / 4$ monopoles with $\lambda / 4$ array spacing. The central element is the normal antenna element and two elements at both sides are the MSEs. A Schottky diode between the MSE monopole and the ground plane is used as the nonlinear impedance for modulation. In the measurement, the normal monopole antenna is connected to a real-time spectrum analyzer (Tektronix RSA3303A) as the RF receiver, which is capable of capturing time-varying RF signal with $15 \mathrm{MHz}$ bandwidth. $5 \mathrm{MHz}$ local signal generated by a signal generator (NF function Synthesizer WF-1966) was applied to the MSEs. Because the same local signal was applied to both of the MSEs for simplicity in this experiment, the array antenna has only two array branches. However, it is possible to increase the number of branches by feeding different frequency to each MSE in the present configurations. The measurement was performed in a non-line-of-sight (NLOS) indoor environment. Several tens of conducting objects with a size of several wavelengths were distributed randomly around the array antenna to realize a Rayleigh fading environment. A log-period array antenna located about 5 meters away from the array antenna radiated $2.4 \mathrm{GHz} \mathrm{CW}$ signal. The MSAA was moved randomly within a range of about 1 meter while the signal with center frequency of $2.4 \mathrm{GHz}$ and bandwidth of $15 \mathrm{MHz}$ was continuously received and stored by the real-time spectrum analyzer.

The signal at $f_{R F}=2.4 \mathrm{GHz}$ and that at $f_{I F}=2.4 \mathrm{GHz}+5 \mathrm{MHz}$ are two received branches corresponding to the normal antenna and the MSEs, 


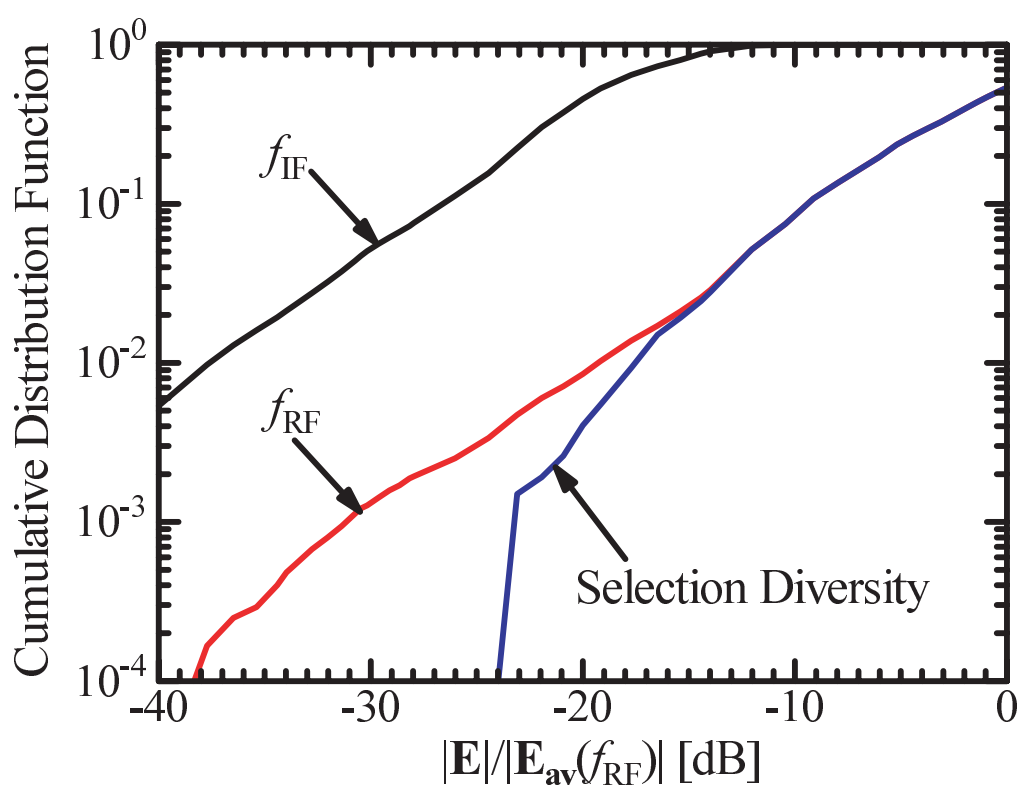

Fig. 3. CDF ofreceived power relative to $f_{R F}$ branch.

respectively. The diversity gain is evaluated when the selection diversity is applied to the two branches. Fig. 3 shows the cumulative distribution function (CDF) of the $f_{R F}$ branch and the $f_{I F}$ branch obtained from 120,000 sampling data recorded in time-domain. The received power of each branches is normalized by the averaged power of the $f_{R F}$ branch. The received level when the selection diversity is introduced is plotted in the same figure. It can be found that the $f_{I F}$ branch is less than the $f_{R F}$ branch by about $18 \mathrm{~dB}$ and the MSAA can provide $2 \mathrm{~dB}$ diversity gain relative to the $f_{R F}$ branch at $1 \%$ of $\mathrm{CDF}$, and $8 \mathrm{~dB}$ diversity gain at $0.1 \%$ of $\mathrm{CDF}$.

\section{Conclusions}

A concept of modulated scattering array antenna based on the modulated scattering technique has been proposed. It has been shown by experimental investigations that a 3 -element modulated scattering array antenna composed of one $\lambda / 4$ monopole antenna and two $\lambda / 4$ modulated scattering monopoles with $\lambda / 4$ array spacing can provide diversity gain up to $8 \mathrm{~dB}$ at $0.1 \%$ of CDF. The array antenna is suitable to the mobile handset applications where the space and the cost are strictly limited because of its simple configurations.

It should be noted that although only the performance of the selection diversity has been investigated, the MSAA can also be used for the adaptive reception. The problem of much lower output level of the $f_{I F}$ branch than that of the $f_{R F}$ branch should be solved to improve the performance of the MSAA, which is the future work of this research.

\section{Acknowledgments}

This research was partly supported by the project of Creation of Innovative Clusters promoted by Ministry of Education, Culture, Sports, Science and Technology of Japan. 